- Supporting Information -

\title{
Host-Guest Silicalite-1 Zeolites: Correlated Disorder and Phase Transition Inhibition by a Small Guest Modification
}

Marco Fabbiani, ${ }^{\text {a }}$ Shadi Al-Nahari, ${ }^{a}$ Laura Piveteau, ${ }^{\mathrm{b}}$ Eddy Dib, ${ }^{\mathrm{a}, \#}$ Vasyl Veremeienko, ${ }^{\mathrm{a}}$ Arnold Gaje, Dan G. Dumitrescu, ${ }^{\mathrm{c}}$ Philippe Gaveau, ${ }^{\mathrm{a}}$ Tzonka Mineva, ${ }^{\mathrm{a}}$ Dominique Massiot, ${ }^{\mathrm{b}}$ Arie van der Lee, ${ }^{\mathrm{d}}$ Julien Haines ${ }^{\mathrm{a}}$ and Bruno Alonso ${ }^{\mathrm{a}, *}$

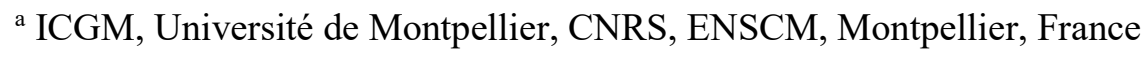

${ }^{\mathrm{b}}$ CNRS, CEMHTI UPR3079, Univ. Orléans, Orléans, France

${ }^{\mathrm{c}}$ Elettra, Sincrotrone Trieste S.C.p.A., Basovizza, Trieste, 34149, Italy

${ }^{\mathrm{d}}$ IEM, Université de Montpellier, CNRS, ENSCM, Montpellier, France

\#E.D.: Laboratoire Catalyse \& Spectrochimie, Normandie Univ, ENSICAEN, UNICAEN, CNRS, 14000 Caen, France

$\S$ A.G.: Department of Chemistry, College of Arts and Sciences, University of the Philippines Visayas, 5023 Miag-ao, Iloilo, Philippines

*E-mail: bruno.alonso@enscm.fr

\section{Content}

page

1. Numbering of Si sites

2. ${ }^{29}$ Si SSNMR: peak widths and transverse relaxation data 3

3. XRPD patterns of TPA-F-HT and TPEA-F-HT 4

4. $\quad{ }^{29} \mathrm{Si} \mathrm{NMR}$ analysis of the F site exchange 5

5. Comparison of ${ }^{29} \mathrm{Si}\left\{{ }^{1} \mathrm{H}\right\} \mathrm{CP}-\mathrm{MAS}$ spectra obtained at low $T$

6. Weighted averages of ${ }^{29} \mathrm{Si}$ NMR chemical shifts at different temperatures 6

7. Comparison of ${ }^{13} \mathrm{C}\left\{{ }^{1} \mathrm{H}\right\}$ CP-MAS spectra at different temperatures 7

8. Comparison of ${ }^{13} \mathrm{C}\left\{{ }^{1} \mathrm{H}\right\}$ CP-MAS spectra of TPA-F-HT and TPEA-F-HT 8

9. DFT optimized structures and stabilization energies 9

10. Complementary ${ }^{29} \mathrm{Si}^{29} \mathrm{Si}\left\{{ }^{1} \mathrm{H}\right\}$ CP-INADEQUATE information 11

11. Assignment of ${ }^{29} \mathrm{Si}$ chemical shifts from INADEQUATE data 12

12. Comparison of atomic displacements of TPA-F-HT and TPEA-F-HT 13

13. ${ }^{29} \mathrm{Si}$ peak widths and atomic displacements of TPA-F-HT 14

14. Comparison of anisotropy displacement parameters of TPA-F-HT and TPEA-F-HT 15

15. SiOSi angle variations between crystal structures at 295 and $100 \mathrm{~K} \quad 16$

$\begin{array}{ll}\text { References } & 17\end{array}$ 


\section{Numbering of Si sites}

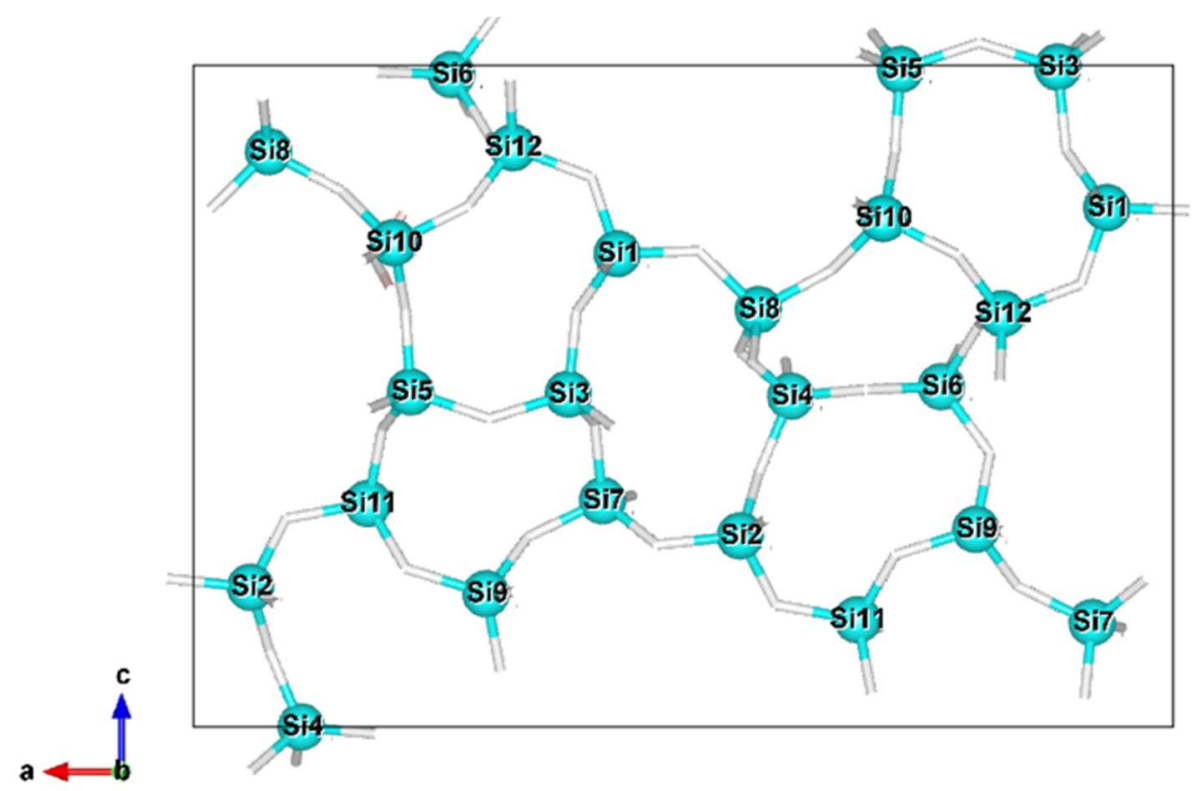

Fig. S1. Si site numbering of the Pnma silicalite-1 zeolites.

The numbering of the Si sites of silicalite-1 zeolites is presented in the figure above (Pnma space group, ac projection). It follows that proposed by Chao et al. ${ }^{1}$ The correspondence between different site numbering is given in the table below.

Table S1. Correspondence between Si sites numbering of Pnma silicalite-1 zeolites.

\begin{tabular}{cc}
\hline Chao et al. ${ }^{1}$ & van Koningsveld et al. ${ }^{2}$ \\
\hline T1 & T5 \\
T2 & T11 \\
T3 & T4 \\
T4 & T10 \\
T5 & T3 \\
T6 & T9 \\
T7 & T7 \\
T8 & T1 \\
T9 & T8 \\
T10 & T2 \\
T11 & T12 \\
T12 & T6 \\
\end{tabular}




\section{2. ${ }^{29}$ Si SSNMR: peak widths and transverse relaxation data}

Table S2. ${ }^{29} \mathrm{Si}$ NMR parameters for the peaks giving separated individual resonances and assigned to T3, T4, T8 and T9 sites in as-synthesized silicalite-1.

\begin{tabular}{|c|c|c|c|c|}
\hline Materials & $\delta\left({ }^{29} \mathrm{Si}\right)(\mathrm{ppm})^{\mathrm{a}}$ & $\mathrm{FWHM}_{(\mathrm{ppm})^{\mathrm{a}}}$ & $T_{2} *(\mathrm{~ms})^{\mathrm{a}}$ & $T_{2}{ }^{\prime}(\mathrm{ms})^{\mathrm{b}}$ \\
\hline \multicolumn{5}{|l|}{ T4 site } \\
\hline TPA-F-HT & -108.4 & 0.62 & 6.5 & 18 \\
\hline TPA-F-SF & -108.2 & 1.40 & 2.9 & 8 \\
\hline TPEA-F-HT & -107.7 & 1.54 & 2.6 & 23 \\
\hline \multicolumn{5}{|l|}{ T8 site } \\
\hline TPA-F-HT & -109.7 & 0.27 & 15 & 48 \\
\hline TPA-F-SF & -109.6 & 0.79 & 5.1 & 27 \\
\hline TPEA-F-HT & -109.2 & 0.79 & 5.1 & 60 \\
\hline \multicolumn{5}{|l|}{ T9 site } \\
\hline TPA-F-HT & -116.8 & 0.37 & 11 & 31 \\
\hline TPA-F-SF & -116.7 & 0.94 & 4.3 & 21 \\
\hline TPEA-F-HT & -116.1 & 1.33 & 3.0 & 25 \\
\hline \multicolumn{5}{|l|}{ T3 site } \\
\hline TPA-F-HT & -117.5 & 0.27 & 15 & 58 \\
\hline TPA-F-SF & -117.4 & 0.59 & 7 & 33 \\
\hline TPEA-F-HT & -117.5 & 0.78 & 5 & 66 \\
\hline
\end{tabular}

${ }^{\text {a }}{ }^{29} \mathrm{Si}$ chemical shifts $\delta\left({ }^{29} \mathrm{Si}\right)$, Full Width at Half Maximum (FWHM) values and related $T_{2} *$ relaxation times were obtained from spectrum fitting (with variable Gaussian/Lorzentzian contributions). The standard errors are within $\pm 0.1 \mathrm{ppm}$ for $\delta$ and \pm 0.05 ppm for FWHM.

b Transverse relaxation times $T_{2}$ ' were measured using a CP-MAS block chained by a spin echo with ${ }^{1} \mathrm{H}$ decoupling. The standard errors are within $\pm 1 \mathrm{~ms}$. 


\section{XRPD patterns of TPA-F-HT and TPEA-F-HT}

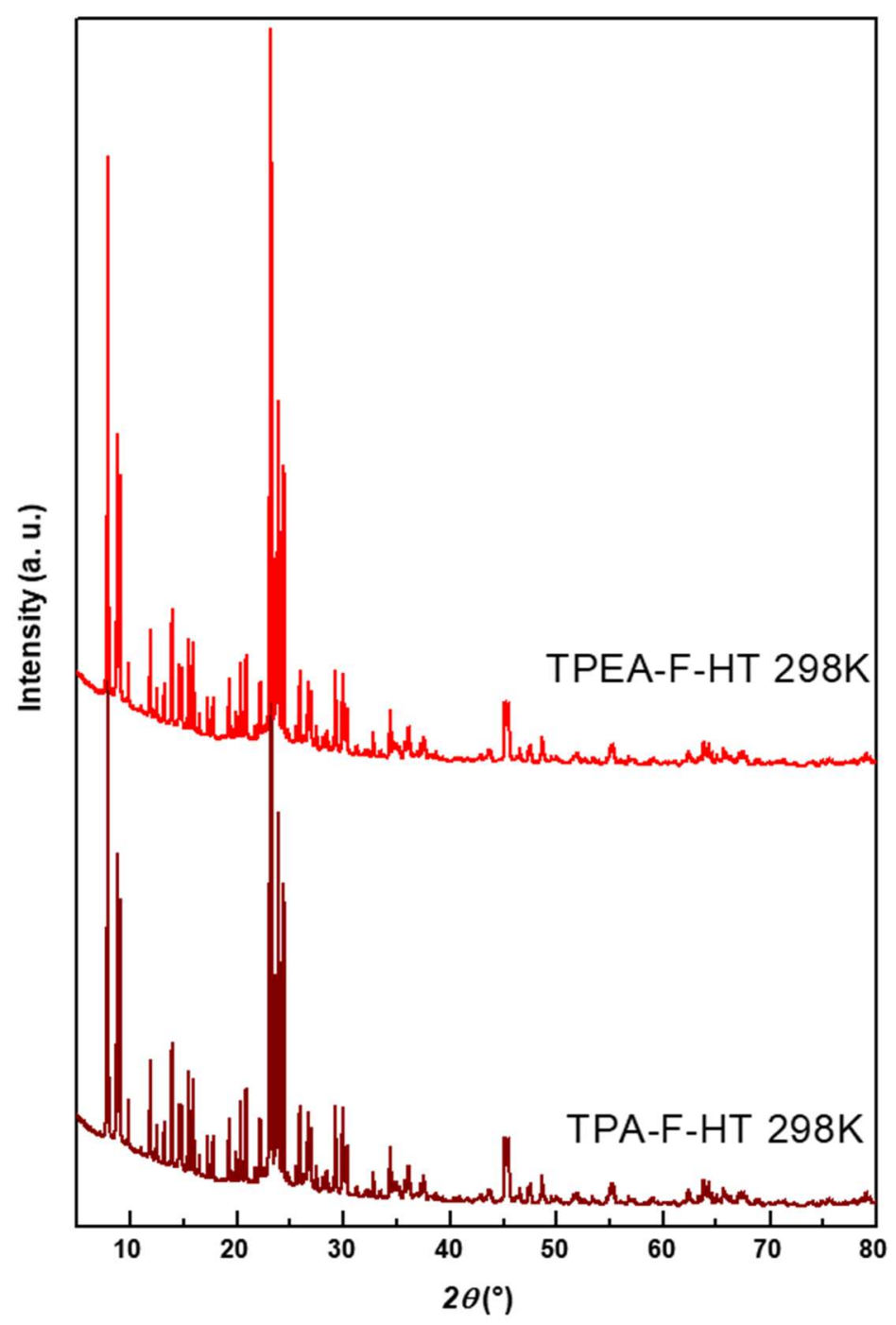

Fig. S2. X-ray powder diffraction patterns of TPA-F-HT and TPEA-F-HT host-guest zeolites. 


\section{4. $\quad{ }^{29} \mathrm{Si}$ NMR analysis of the $F$ site exchange}
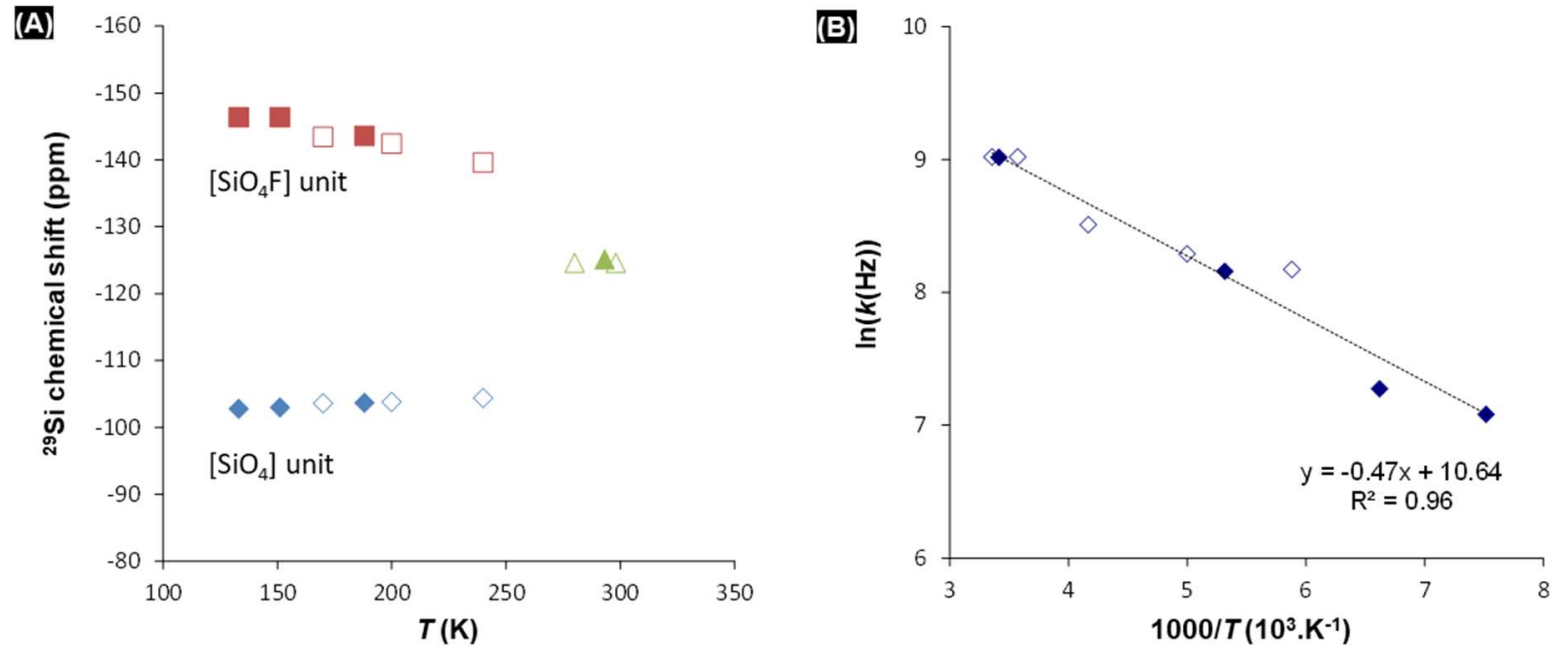

Fig. S3. Analysis of the F exchange rate in the $\left[4^{1} 5^{2} 6^{2}\right]$ cages of TPA-F-HT. (A) Evolution of the ${ }^{29}$ Si chemical shits related to T6 sites as a function of temperature. (B) Arrhenius plot of the F exchange rate $k$. The exchange rate is calculated from the equation $\Delta \omega(T)=\left(\left(\Delta \omega_{0}\right)^{2}-4 k^{2}\right)^{1 / 2}$ related to a simple two-sites exchange model where $\omega_{0}$ values correspond to the precession frequencies of $\left[\mathrm{SiO}_{4} \mathrm{~F}\right]$ and $\left[\mathrm{SiO}_{4}\right]$ units at the lowest $T$. From this plot, the activation energy for $\mathrm{F}$ exchange is estimated to be: $E_{A}=3.9 \mathrm{~kJ} \cdot \mathrm{mol}^{-1}$. Empty symbols correspond to the experimental data of Koller et al., ${ }^{3}$ and filled symbols to this work 


\section{Comparison of ${ }^{29} \mathrm{Si}\left\{{ }^{1} \mathrm{H}\right\}$ CP-MAS spectra obtained at low $T$}

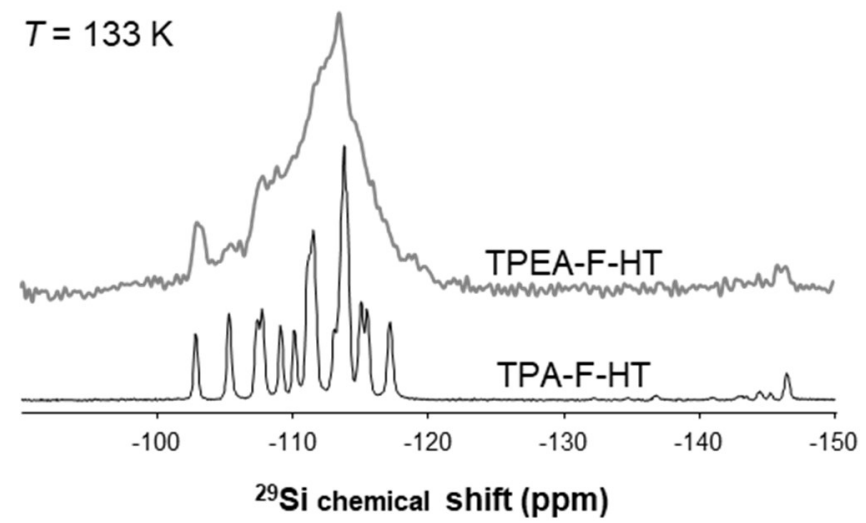

Fig. S4. ${ }^{29} \mathrm{Si}\left\{{ }^{1} \mathrm{H}\right\}$ CP-MAS spectra of TPA-F-HT and TPEA-F-HT host-guest zeolites recorded at $T=133 \mathrm{~K}\left(v_{0}\left[{ }^{29} \mathrm{Si}\right] \equiv 119.2 \mathrm{MHz}, v_{M A S}=3.5 \mathrm{kHz}\right)$.

\section{Weighted averages of ${ }^{29} \mathrm{Si}$ NMR chemical shifts at different temperatures}

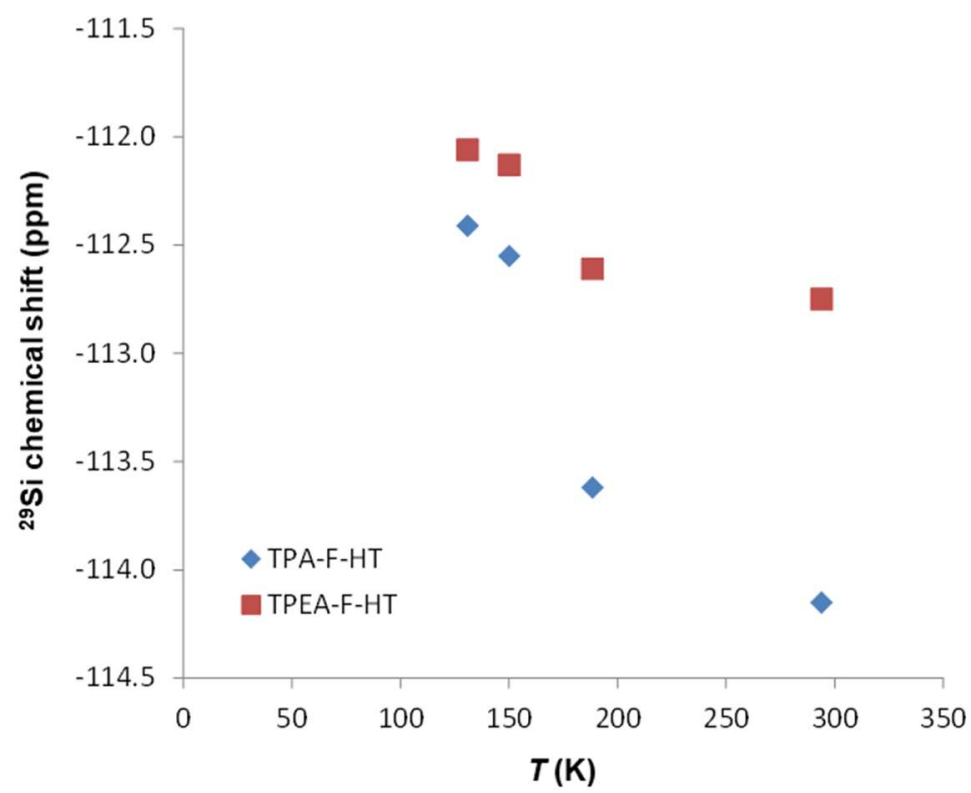

Fig. S5. Evolution of the weighted averages of ${ }^{29} \mathrm{Si}$ chemical shifts of TPA-F-HT and TPEA-F-HT host-guest zeolites as a function of temperature. 


\section{Comparison of ${ }^{13} \mathrm{C}\left\{{ }^{1} \mathrm{H}\right\} \mathrm{CP}-\mathrm{MAS}$ spectra at different temperatures}
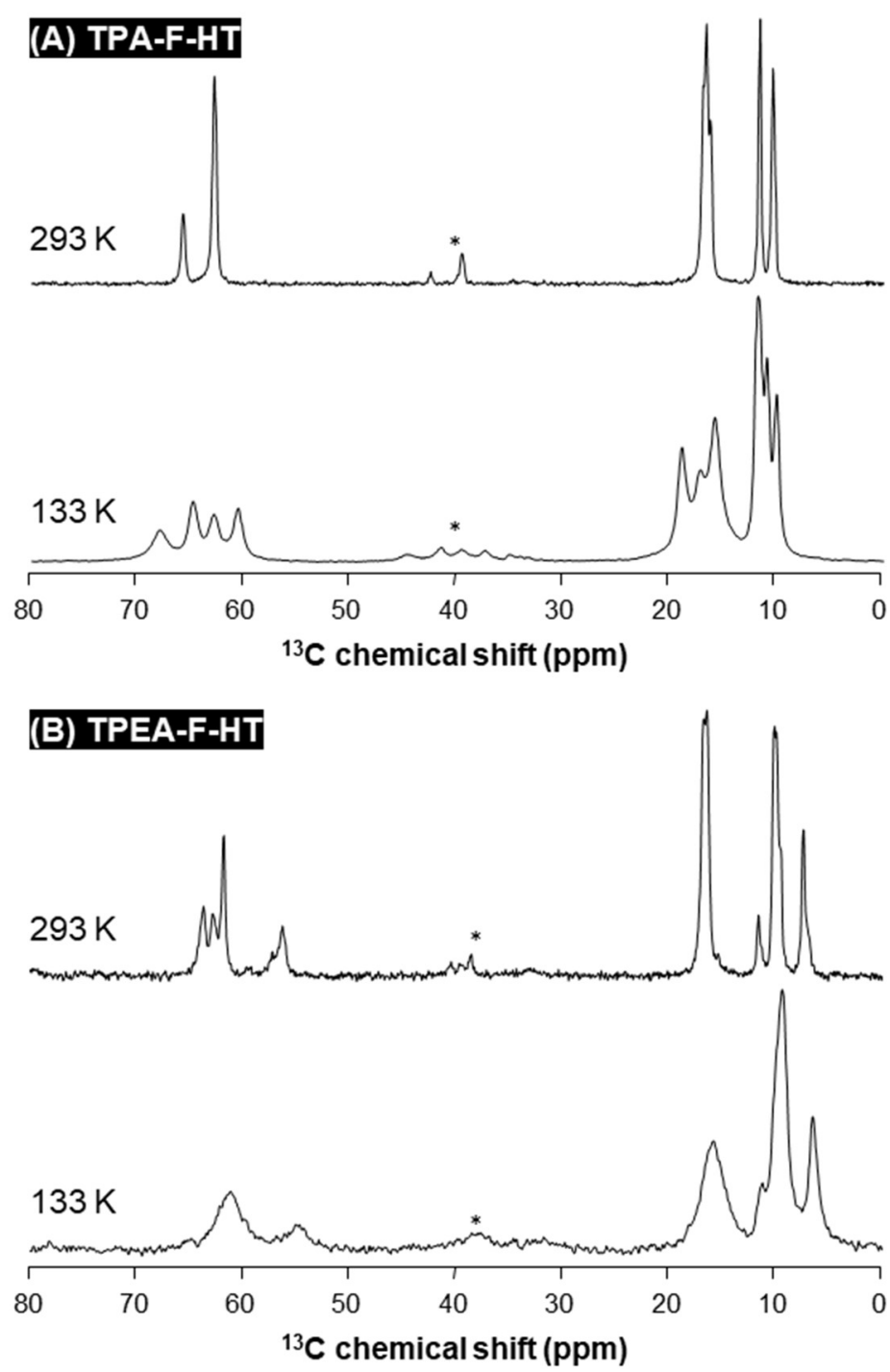

Fig. S6. ${ }^{13} \mathrm{C}\left\{{ }^{1} \mathrm{H}\right\}$ CP-MAS spectra of (A) TPA-F-HT and (B) TPEA-F-HT host-guest zeolites recorded at $T=$ $293 \mathrm{~K}$ and $133 \mathrm{~K}\left(v_{0}\left[{ }^{13} \mathrm{C}\right]=150.8 \mathrm{MHz}, v_{M A S}=3.5 \mathrm{kHz}\right)$. Asterisks refer to spinning sidebands' peaks. 


\section{Comparison of ${ }^{13} \mathrm{C}\left\{{ }^{1} \mathrm{H}\right\}$ CP-MAS spectra of TPA-F-HT and TPEA-F-HT}
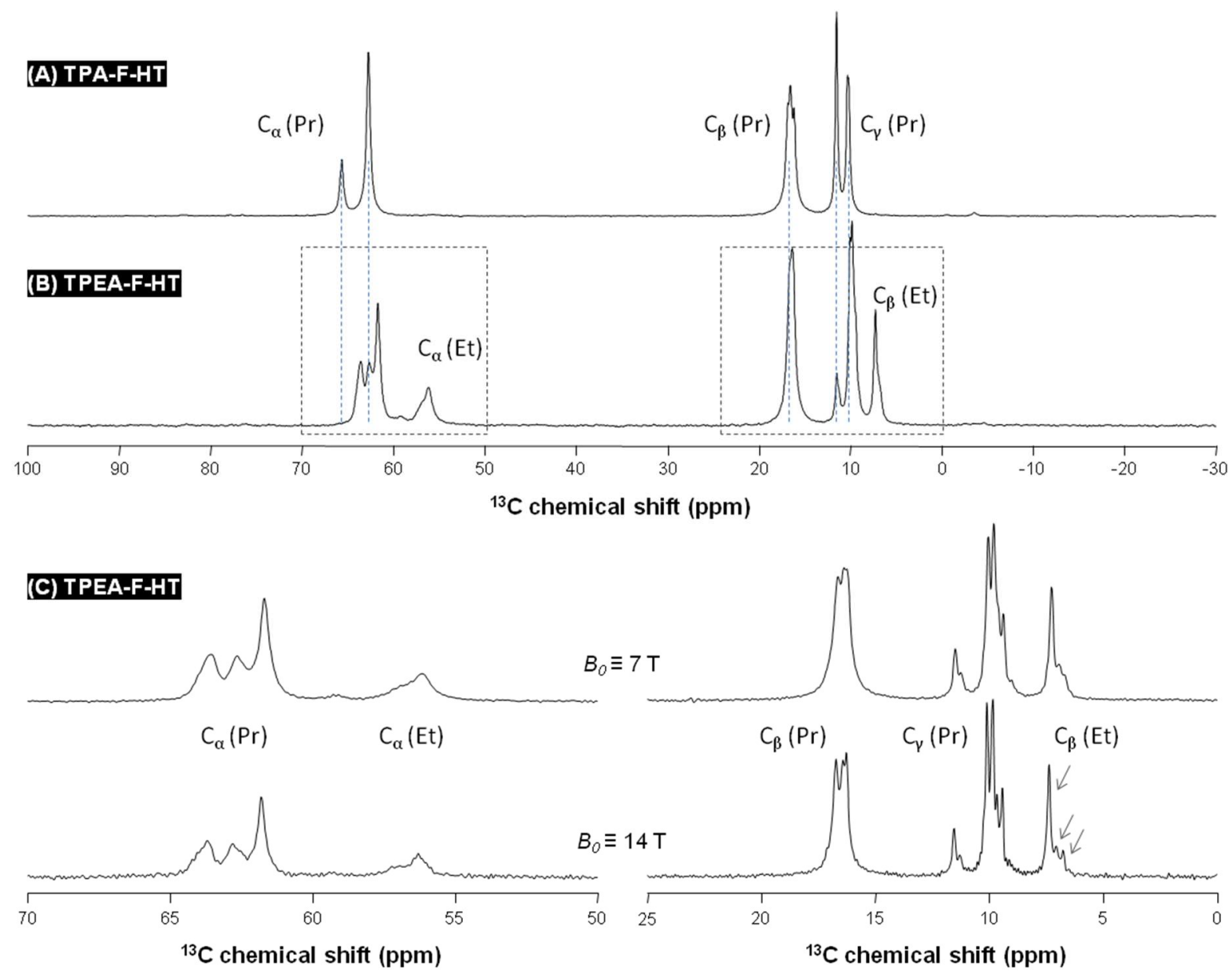

Fig. S7. ${ }^{13} \mathrm{C}\left\{{ }^{1} \mathrm{H}\right\}$ CP-MAS spectra at $T=293 \mathrm{~K}$ of (A) TPA-F-HT and (B) TPEA-F-HT host-guest zeolites $\left(v_{0}\left[{ }^{13} \mathrm{C}\right]=75.5 \mathrm{MHz}, v_{M A S}=5 \mathrm{kHz}\right)$. (C) Comparison of ${ }^{13} \mathrm{C}\left\{{ }^{1} \mathrm{H}\right\}$ CP-MAS spectra of TPEA-F-HT at two different magnetic fields (zoomed areas). The arrows show the different small peaks observed for $\mathrm{C}_{\beta}(\mathrm{Et})$ and related to different Et orientations (see text). 


\section{DFT optimized structures and stabilization energies}
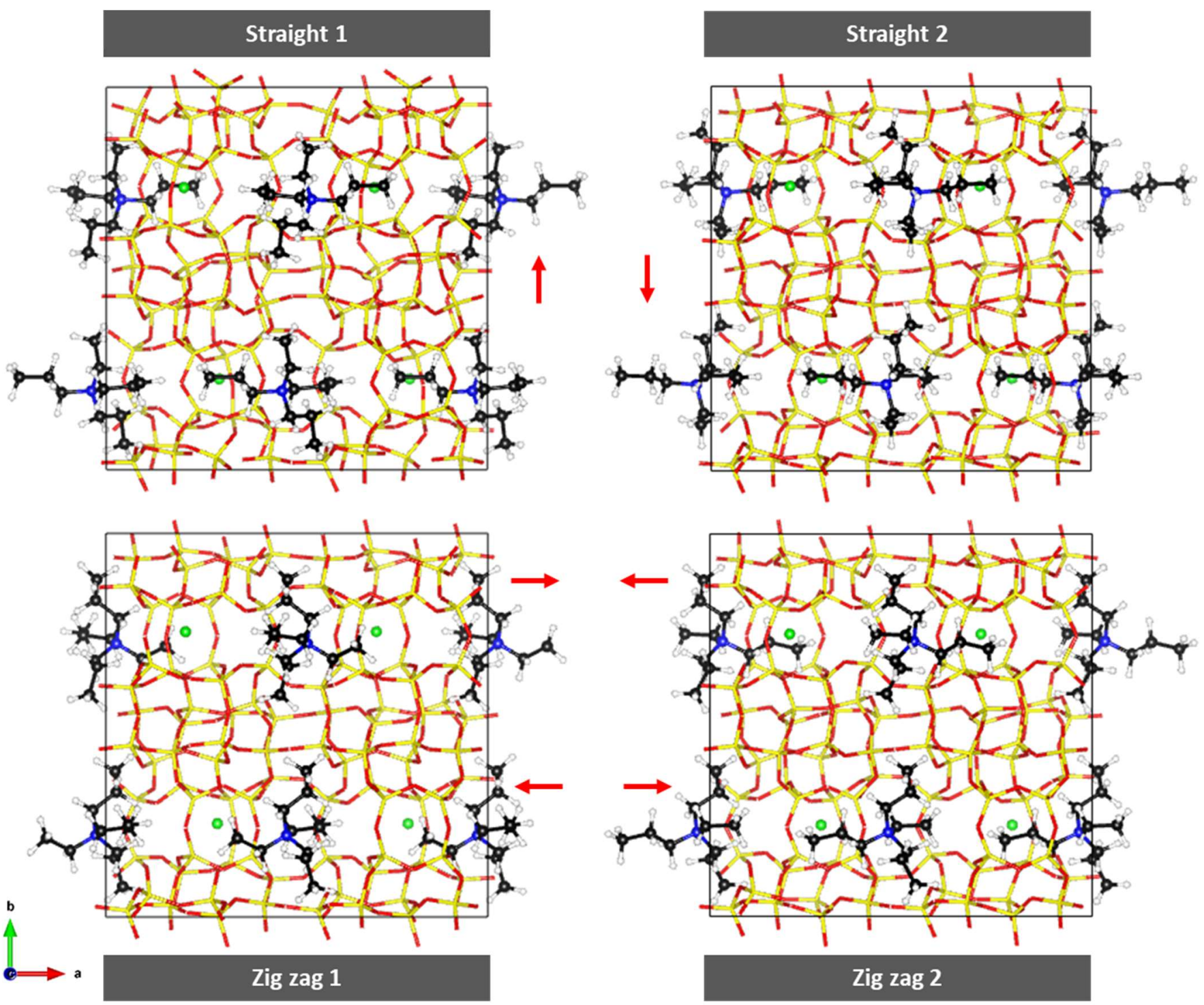

Fig. S8. DFT-D geometrically optimized models of the four TPEA-F-silicalite-1 host-guest zeolites, with $P n 2_{1} a$ space group and different locations for the ethyl arm, presented in an ab projection. The directions of the ethyl arms are shown by red arrows. The atoms and bonds are represented using balls and sticks following CPK notations ( $\mathrm{H}, \mathrm{C}, \mathrm{N}, \mathrm{O}, \mathrm{F}$ and $\mathrm{Si}$ in white, black, blue, red, green and yellow respectively). Si and $\mathrm{O}$ atoms are omitted for the sake of clarity.

The related stabilization ernergies per OSDA $E_{\text {stab }}$ are presented in the table below. For each model, this energy is calculated from the energies of the optimized host-guest zeolite $E_{z e o}$, the optimized silica framework alone $E_{F W}$, the optimized OSDA alone $E_{O S D A}$, and the optimized $\mathrm{F}$ alone $E_{F}$ following: $E_{\text {stab }}=\left(E_{z e o}-E_{F W}-4 \cdot E_{O S D A}-4 . E_{F}\right) / 4$ 
Table S3. Stabilization energies of DFT geometrically optimized models of TPA-F-silicalite-1 and the four TPEA-F-silicalite-1 host-guest zeolites presented above.

\begin{tabular}{ll}
\hline Optimized model & Stabilization energy $\left(\mathrm{kJ} \cdot \mathrm{mol}^{-1}\right)$ \\
\hline TPA-F (reference) & -1085 \\
ETPA-F-straight 1 & -1105 \\
ETPA-F-straight 2 & -1079 \\
ETPA-F-zig zag 1 & -1076 \\
ETPA-F-zig zag 2 & -1084 \\
\hline
\end{tabular}



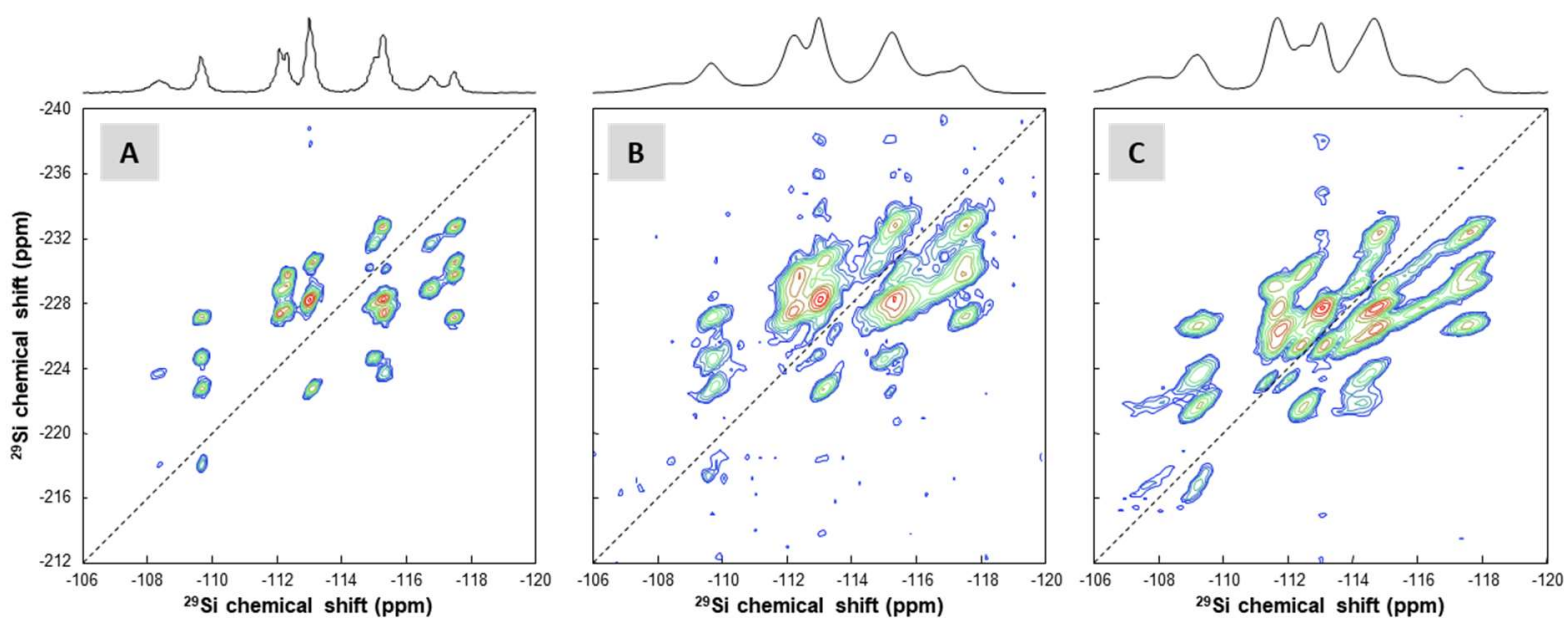

Fig. S9. 2D DQ-SQ ${ }^{29} \mathrm{Si}^{2}{ }^{29} \mathrm{Si}\left\{{ }^{1} \mathrm{H}\right\}$ CP-INADEQUATE-MAS spectra of host-guest zeolites: (A) TPA-F-HT, (B) TPA-F-SF, (C) TPEA-F-HT. The contour lines of the three 2D spectra have been obtained with identical graphical parameters.
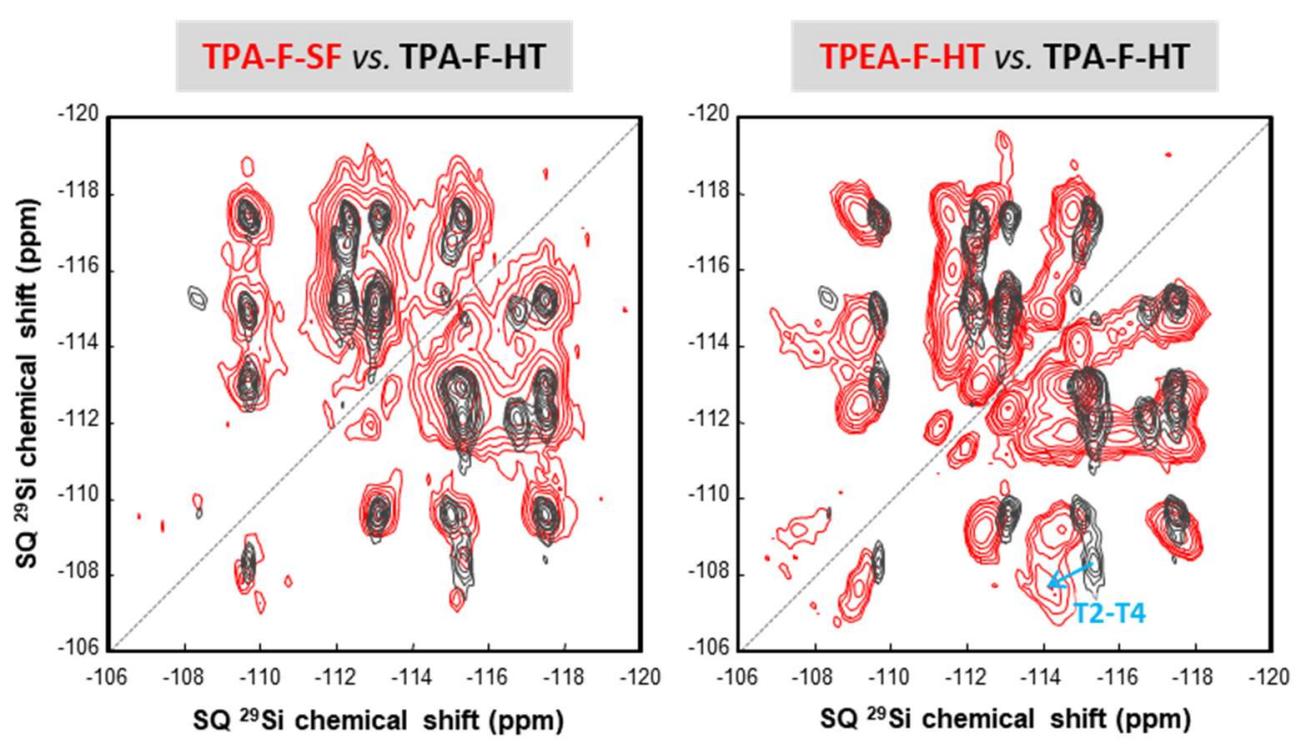

Fig. S10. Comparison of 2D SQ-SQ ${ }^{29} \mathrm{Si}^{29}{ }^{29} \mathrm{Si}\left\{{ }^{1} \mathrm{H}\right\}$ CP-INADEQUATE-MAS spectra of host-guest zeolites. The contour lines of the three 2D spectra have been obtained with identical graphical parameters. The blue arrow exemplifies the variations in chemical shift observed for the cross-peaks when replacing TPA by TPEA in the HT syntheses (here for the cross-peak related to the pair of bound T2 and T4 Si sites). 


\section{Assignment of ${ }^{29} \mathrm{Si}$ chemical shifts from INADEQUATE data}

Table S4. ${ }^{29} \mathrm{Si}$ chemical shifts of the 12 crystal Si sites in TPA-F-HT and TPEA-F-HT host-guest zeolites (Pnma space group, Chao numbering).

\begin{tabular}{ccc}
\hline & \multicolumn{2}{c}{$\delta\left({ }^{29} \mathrm{Si}\right)(\mathrm{ppm})^{\mathrm{a}}$} \\
Crystal Si site & TPA-F-HT & TPEA-F-HT \\
\hline T4 & -108.4 & -107.7 \\
T8 & -109.7 & -109.2 \\
T11 & -112.1 & -111.8 \\
T7 & -112.3 & -111.6 \\
T12 & -113.0 & -113.0 \\
T1 & -113.1 & -112.5 \\
T10 & -114.9 & -114.3 \\
T5 & -115.2 & -114.8 \\
T2 & -115.4 & -114.1 \\
T9 & -116.8 & -116.1 \\
T3 & -117.5 & -117.5 \\
T6 & -125.2 & -124.7 \\
\hline
\end{tabular}

${ }^{\text {a }}{ }^{29} \mathrm{Si}$ chemical shifts were obtained from fitting 1D and $2 \mathrm{D}{ }^{29} \mathrm{Si}$ NMR data after assignment of $2 \mathrm{D}^{29} \mathrm{Si}^{29}{ }^{29} \mathrm{Si}\left\{{ }^{1} \mathrm{H}\right\}$ CP-INADEQUATE spectra. The standard errors are within $\pm 0.1 \mathrm{ppm}$. 


\section{Comparison of atomic displacements of TPA-F-HT and TPEA-F-HT}

Table S5. Mean squared atomic displacements $U_{i s o}$ of Si, O and F atoms in TPA-F-HT and TPEA-F-HT host-guest zeolites from the crystal structures determined at $T=295 \mathrm{~K}$.

\begin{tabular}{|c|c|c|}
\hline $\begin{array}{c}\text { Atom sites } \\
\text { Chao numbering }\end{array}$ & $\begin{array}{c}U_{i s o}\left(\AA^{2}\right) \\
\text { TPA-F-HT }(295 \mathrm{~K}) \\
\end{array}$ & $\begin{array}{c}U_{i s o}\left(\AA^{2}\right) \\
\text { TPEA-F-HT }(295 \mathrm{~K})\end{array}$ \\
\hline Si1 & 0.015 & 0.020 \\
\hline $\mathrm{Si} 2$ & 0.016 & 0.021 \\
\hline $\mathrm{Si} 3$ & 0.015 & 0.020 \\
\hline $\mathrm{Si} 4$ & 0.018 & 0.023 \\
\hline $\mathrm{Si} 5$ & 0.016 & 0.021 \\
\hline Si6 & 0.021 & 0.026 \\
\hline $\mathrm{Si} 7$ & 0.016 & 0.019 \\
\hline $\mathrm{Si} 8$ & 0.016 & 0.020 \\
\hline $\mathrm{Si} 9$ & 0.017 & 0.021 \\
\hline Si10 & 0.018 & 0.022 \\
\hline Sil1 & 0.017 & 0.021 \\
\hline Si12 & 0.017 & 0.022 \\
\hline Mean Si & 0.017 & 0.021 \\
\hline $\mathrm{O} 1$ & 0.029 & 0.034 \\
\hline $\mathrm{O} 2$ & 0.036 & 0.041 \\
\hline $\mathrm{O} 3$ & 0.044 & 0.046 \\
\hline $\mathrm{O} 4$ & 0.036 & 0.041 \\
\hline O5 & 0.047 & 0.053 \\
\hline O6 & 0.036 & 0.040 \\
\hline $\mathrm{O} 7$ & 0.041 & 0.046 \\
\hline O8 & 0.036 & 0.040 \\
\hline O9 & 0.042 & 0.044 \\
\hline $\mathrm{O} 10$ & 0.040 & 0.045 \\
\hline $\mathrm{O} 11$ & 0.050 & 0.055 \\
\hline $\mathrm{O} 12$ & 0.041 & 0.047 \\
\hline $\mathrm{O} 13$ & 0.068 & 0.074 \\
\hline $\mathrm{O} 14$ & 0.036 & 0.041 \\
\hline O15 & 0.031 & 0.036 \\
\hline O16 & 0.054 & 0.059 \\
\hline O17 & 0.041 & 0.046 \\
\hline O18 & 0.026 & 0.030 \\
\hline O19 & 0.031 & 0.034 \\
\hline O20 & 0.034 & 0.041 \\
\hline $\mathrm{O} 21$ & 0.038 & 0.043 \\
\hline $\mathrm{O} 22$ & 0.030 & 0.035 \\
\hline $\mathrm{O} 23$ & 0.037 & 0.040 \\
\hline $\mathrm{O} 24$ & 0.036 & 0.044 \\
\hline $\mathrm{O} 25$ & 0.036 & 0.041 \\
\hline $\mathrm{O} 26$ & 0.031 & 0.035 \\
\hline $\mathrm{F} 1$ & 0.148 & 0.107 \\
\hline
\end{tabular}




\section{3. ${ }^{29} \mathrm{Si}$ peak widths and atomic displacements of TPA-F-HT}

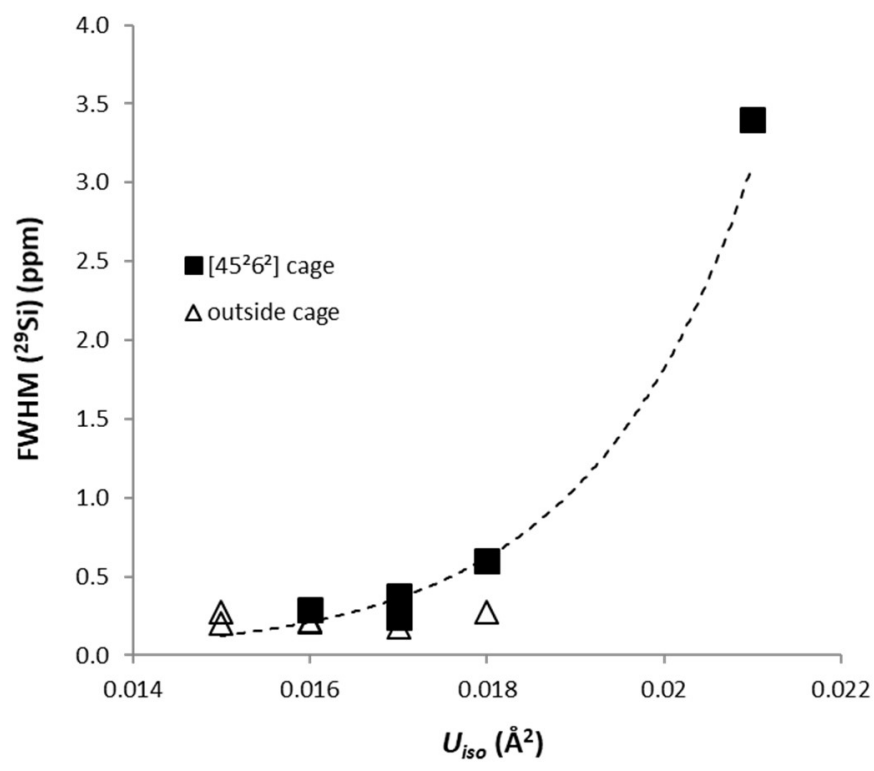

Fig. S11. Variations of the full width at half maximum of ${ }^{29} \mathrm{Si}$ peaks as a function of the mean squared atomic displacements $U_{i s o}$ from NMR and XRD data obtained for TPA-F-HT host-guest zeolite at ambient temperature ( $T=293$ and $295 \mathrm{~K}$ respectively). Filled squares correspond to Si sites in the $\left[4^{1} 5^{2} 6^{2}\right]$ cage containing F, empty triangles correspond to the other Si sites. The line is only a guide to eyes. 


\section{Comparison of anisotropy displacement parameters of TPA-F-HT and TPEA-F-HT}

Table S6. Figure of merits of $\mathrm{Si}, \mathrm{O}$ and $\mathrm{F}$ atoms in TPA-F-HT and TPEA-F-HT host-guest zeolites from the anisotropy displacement parameters of crystal structures determined at $T=295 \mathrm{~K}$.

\begin{tabular}{|c|c|c|}
\hline $\begin{array}{c}\text { Atom sites } \\
\text { Chao numbering }\end{array}$ & $\begin{array}{c}\text { Figure of Merit (FOM) } \\
\text { TPA-F-HT } v s . \text { TPEA-F-HT (295K) }\end{array}$ & $\begin{array}{c}\text { Figure of Merit (FOM) } \\
\text { 100K vs. 295K (TPEA-F-HT) }\end{array}$ \\
\hline Si 1 & 0.10 & 0.15 \\
\hline Si 2 & 0.14 & 0.17 \\
\hline Si 3 & 0.12 & 0.09 \\
\hline $\mathrm{Si} 4$ & 0.08 & 0.16 \\
\hline Si 5 & 0.10 & 0.14 \\
\hline Si 6 & 0.05 & 0.14 \\
\hline Si 7 & 0.15 & 0.15 \\
\hline Si 8 & 0.14 & 0.16 \\
\hline Si 9 & 0.08 & 0.09 \\
\hline Si 10 & 0.09 & 0.04 \\
\hline Si 11 & 0.07 & 0.10 \\
\hline Si 12 & 0.08 & 0.11 \\
\hline Mean Si & 0.10 & 0.13 \\
\hline $\mathrm{O} 1$ & 0.03 & 0.11 \\
\hline $\mathrm{O} 2$ & 0.04 & 0.17 \\
\hline $\mathrm{O} 3$ & 0.06 & 0.08 \\
\hline $\mathrm{O} 4$ & 0.02 & 0.08 \\
\hline O5 & 0.03 & 0.08 \\
\hline O6 & 0.04 & 0.07 \\
\hline $\mathrm{O} 7$ & 0.03 & 0.07 \\
\hline O8 & 0.03 & 0.17 \\
\hline O9 & 0.04 & 0.11 \\
\hline $\mathrm{O} 10$ & 0.05 & 0.15 \\
\hline O11 & 0.05 & 0.12 \\
\hline $\mathrm{O} 12$ & 0.04 & 0.09 \\
\hline O13 & 0.03 & 0.20 \\
\hline O14 & 0.03 & 0.11 \\
\hline O15 & 0.02 & 0.09 \\
\hline O16 & 0.04 & 0.12 \\
\hline O17 & 0.04 & 0.14 \\
\hline $\mathrm{O} 18$ & 0.03 & 0.09 \\
\hline O19 & 0.02 & 0.07 \\
\hline $\mathrm{O} 20$ & 0.04 & 0.11 \\
\hline $\mathrm{O} 21$ & 0.04 & 0.03 \\
\hline $\mathrm{O} 22$ & 0.05 & 0.13 \\
\hline $\mathrm{O} 23$ & 0.04 & 0.15 \\
\hline $\mathrm{O} 24$ & 0.02 & 0.09 \\
\hline $\mathrm{O} 25$ & 0.03 & 0.14 \\
\hline $\mathrm{O} 26$ & 0.04 & 0.10 \\
\hline F1 & 0.11 & 0.33 \\
\hline
\end{tabular}




\section{SiOSi angle variations between crystal structures at 295 and $100 \mathrm{~K}$}

Table S7. SiOSi angle variations of TPA-F-HT and TPEA-F-HT host-guest zeolites from crystal structures determined at $T=295$ and $100 \mathrm{~K}$.

\begin{tabular}{|c|c|c|c|c|c|c|}
\hline Si sites $(P n m a)$ & $\begin{array}{c}\text { TPA-F-HT } \\
T=295 \mathrm{~K} \\
(\text { Pnma }) \\
\end{array}$ & $\begin{array}{c}\text { SiOSi }\left({ }^{\circ}\right) \\
T=100 \mathrm{~K} \\
\left(P 2_{1} / n\right)\end{array}$ & Difference & $\begin{array}{c}\text { TPEA-F-HT } \\
T=295 \mathrm{~K} \\
(\text { Pnma }) \\
\end{array}$ & $\begin{array}{c}\mathrm{SiOSi}\left({ }^{\circ}\right) \\
T=100 \mathrm{~K} \\
(\text { Pnma })\end{array}$ & Difference \\
\hline $\mathrm{T} 1, \mathrm{~T} 2$ & 167.5 & 156.5 & 11.1 & 166.1 & 157.4 & 8.7 \\
\hline $\mathrm{T} 1, \mathrm{~T} 2$ & 167.5 & 162.3 & 5.2 & & & \\
\hline $\mathrm{T} 1, \mathrm{~T} 3$ * & 156.7 & 155.6 & 1.1 & 155.8 & 154.2 & 1.6 \\
\hline $\mathrm{T} 1, \mathrm{~T} 3 *$ & 156.7 & 154.3 & 2.5 & & & \\
\hline $\mathrm{T} 1, \mathrm{~T} 8$ * & 147.2 & 147.4 & -0.2 & 147.0 & 144.7 & 2.3 \\
\hline $\mathrm{T} 1, \mathrm{~T} 8$ * & 147.2 & 145.3 & 1.9 & & & \\
\hline $\mathrm{T} 1, \mathrm{~T} 12$ & 148.2 & 143.4 & 4.8 & 147.4 & 141.9 & 5.5 \\
\hline $\mathrm{T} 1, \mathrm{~T} 12$ & 148.2 & 142.8 & 5.4 & & & \\
\hline $\mathrm{T} 2, \mathrm{~T} 4$ * & 163.5 & 158.8 & 4.7 & 162.1 & 157.5 & 4.6 \\
\hline $\mathrm{T} 2, \mathrm{~T} 4$ * & 163.5 & 158.8 & 4.7 & & & \\
\hline $\mathrm{T} 2, \mathrm{~T} 7$ & 149.7 & 146.9 & 2.8 & 149.1 & 143.2 & 5.8 \\
\hline $\mathrm{T} 2, \mathrm{~T} 7$ & 149.7 & 145.3 & 4.3 & & & \\
\hline $\mathrm{T} 2, \mathrm{~T} 11$ & 152.0 & 143.6 & 8.4 & 150.5 & 142.0 & 8.5 \\
\hline $\mathrm{T} 2, \mathrm{~T} 11$ & 152.0 & 144.9 & 7.1 & & & \\
\hline $\mathrm{T} 3, \mathrm{~T} 5$ & 176.7 & 178.3 & -1.6 & 176.0 & 171.0 & 5.0 \\
\hline $\mathrm{T} 3, \mathrm{~T} 5$ & 176.7 & 166.7 & 10.0 & & & \\
\hline $\mathrm{T} 3, \mathrm{~T}^{*}$ & 148.7 & 148.0 & 0.7 & 148.6 & 148.0 & 0.6 \\
\hline $\mathrm{T} 3, \mathrm{~T}^{*}$ & 148.7 & 148.5 & 0.3 & & & \\
\hline T3,T8* & 168.2 & 165.9 & 2.3 & 168.0 & 166.5 & 1.5 \\
\hline $\mathrm{T} 3, \mathrm{~T} 8$ * & 168.2 & 168.8 & -0.6 & & & \\
\hline T4,T4 & 142.8 & 140.5 & 2.3 & 143.2 & 140.7 & 2.5 \\
\hline T4,T6 & 147.8 & 143.5 & 4.3 & 146.5 & 140.0 & 6.6 \\
\hline $\mathrm{T} 4, \mathrm{~T} 6$ & 147.8 & 139.2 & 8.6 & & & \\
\hline T4,T8 * & 145.3 & 140.0 & 5.3 & 144.5 & 137.8 & 6.7 \\
\hline $\mathrm{T} 4, \mathrm{~T} 8$ * & 145.3 & 139.0 & 6.3 & & & \\
\hline T5,T10 & 150.5 & 150.6 & -0.1 & 150.6 & 151.2 & -0.6 \\
\hline $\mathrm{T} 5, \mathrm{~T} 10$ & 150.5 & 153.3 & -2.8 & & & \\
\hline T5,T11 & 145.6 & 143.2 & 2.4 & 144.3 & 142.1 & 2.2 \\
\hline $\mathrm{T5}, \mathrm{T} 11$ & 145.6 & 142.3 & 3.3 & & & \\
\hline $\mathrm{T} 5, \mathrm{~T} 12$ & 161.9 & 157.2 & 4.8 & 161.0 & 155.3 & 5.8 \\
\hline $\mathrm{T} 5, \mathrm{~T} 12$ & 161.9 & 159.8 & 2.2 & & & \\
\hline T6,T6 & 143.5 & 136.1 & 7.4 & 141.7 & 133.7 & 8.1 \\
\hline T6,T9 & 155.8 & 154.4 & 1.4 & 155.3 & 152.9 & 2.4 \\
\hline T6,T9 & 155.8 & 155.2 & 0.6 & & & \\
\hline $\mathrm{T} 6, \mathrm{~T} 12$ & 144.1 & 147.1 & -3.0 & 144.7 & 146.6 & -1.9 \\
\hline $\mathrm{T} 6, \mathrm{~T} 12$ & 144.1 & 143.4 & 0.7 & & & \\
\hline T7,T7 & 152.4 & 148.8 & 3.5 & 152.3 & 146.9 & 5.5 \\
\hline $\mathrm{T7}, \mathrm{T9}$ & 154.3 & 150.0 & 4.3 & 153.8 & 150.0 & 3.8 \\
\hline T7,T9 & 154.3 & 152.1 & 2.2 & & & \\
\hline $\mathrm{T} 8, \mathrm{~T} 10$ & 148.8 & 145.7 & 3.2 & 148.1 & 145.3 & 2.8 \\
\hline $\mathrm{T} 8, \mathrm{~T} 10$ & 148.8 & 145.3 & 3.5 & & & \\
\hline T9,T10 & 173.2 & 158.2 & 14.9 & 171.6 & 155.6 & 16.0 \\
\hline $\mathrm{T9,T10}$ & 173.2 & 160.4 & 12.8 & & & \\
\hline T9,T11 & 170.1 & 164.2 & 5.9 & 169.2 & 158.7 & 10.6 \\
\hline T9,T11 & 170.1 & 161.2 & 8.9 & & & \\
\hline $\mathrm{T} 10, \mathrm{~T} 12$ & 159.3 & 161.6 & -2.3 & 159.7 & 161.5 & -1.8 \\
\hline $\mathrm{T} 10, \mathrm{~T} 12$ & 159.3 & 160.1 & -0.8 & & & \\
\hline $\mathrm{T} 11, \mathrm{~T} 11$ & 148.2 & 151.4 & -3.2 & 148.4 & 151.0 & -2.6 \\
\hline Mean values & 155.4 & 151.8 & 3.6 & 154.1 & 149.8 & 4.2 \\
\hline
\end{tabular}

\footnotetext{
* Si sites presenting a correlated disorder in TPEA-F-HT from ${ }^{29} \mathrm{Si}$ NMR.
} 


\section{References}

1. Chao, K. J.; Lin, J. C.; Wang, Y.; Lee, G. H., Single-Crystal Structure Refinement of TPA ZSM-5 Zeolite. Zeolites 1986, 6, 35-38.

2. van Koningsveld, H.; van Bekkum, H.; Jansen, J. C., On the Location and Disorder of the Tetrapropylammonium (TPA) Ion in Zeolite ZSM-5 with Improved Framework Accuracy. Acta Cryst. B 1987, 43, 127-132.

3. Koller, H.; Wolker, A.; Villaescusa, L. A.; Diaz-Cabanas, M. J.; Valencia, S.; Camblor, M. A., Five-Coordinate Silicon in High-Silica Zeolites. J. Am. Chem. Soc. 1999, 121, 3368-3376. 